\title{
Using speciation diagrams to improve synthesis of magnetic nanosorbents for environmental applications
}

\author{
A F C CAMPOS*, R AQUINO, T A P G COTTA ${ }^{\dagger}, \mathbf{F}$ A TOURINHO $^{\dagger}$ and \\ J DEPEYROT ${ }^{\dagger \dagger}$ \\ Faculdade UnB - Planaltina, Universidade de Brasília, 73300-000, Brasília - DF, Brazil \\ †Instituto de Química, Universidade de Brasília, 70919-900, Brasília - DF, Brazil \\ ${ }^{\dagger \dagger}$ Instituto de Física, Universidade de Brasília, 70919-900, Brasília - DF, Brazil
}

MS received 24 March 2010

\begin{abstract}
This work addresses environmental applications of magnetic nanoparticles. We highlight the chemical design of filtration aids based on magnetic nanoferrites coated with specific ligands potentially used in magnetic separation of pollutants from water. From electrochemical measurements, we determined the concentration of the surface sites in function of $\mathrm{pH}$ for the precursor magnetic nanoparticles. Then, coupling the speciation diagrams of the precursor nanoferrite particle surface with that of the specific ligand, it was possible to provide a theoretical prediction of the optimal $\mathrm{pH}$ for particle surface-ligand complexation, leading to advances in nanosorbents developing.
\end{abstract}

Keywords. Magnetic nanosorbents; surface charge density; environmental applications.

\section{Introduction}

Currently many of the promising advances in nanoscale science and engineering involve the environment. In particular, nanotechnologies play a very important role in recent efforts to develop better processes and methods for remediation and monitoring of contaminated systems with hazardous substances (Tratnyek and Johnson 2006). In this context, materials as nanosorbents, nanocatalysts, bioactive nanoparticles and nanostructured catalytic membranes have been increasingly applied to groundwater, wastewater and drinking water remediation (Savage and Diallo 2005). In the case of magnetic nanosized particles, the procedure of magnetic filtration has been used as an interesting tool concerning the process of organic chemical pollutants removal from water (Mayoa et al 2007). However, since most of these contaminants are not magnetic, it is necessary to develop nanostructured filtration aids to adsorb the pollution agents allowing their magnetic separation. These magnetic nanosorbents have been prepared by coating magnetic nanoparticles with specific ligands that present chemical affinity with the contaminants (Apblett et al 2001). Regarding the petrochemicals removal from water, iron oxides nanocomposites based on magnetite nanoparticles coated with polydimethylsiloxane (PDMS) and polyoctylmethylsiloxane (POMS) have been used to enhance their adsorption

\footnotetext{
*Author for correspondence (relex@unb.br)
}

of hydrocarbons (Apblett et al 2001; Konieczny et al 2007). In this process, carboxylate-functional PDMS or POMS are added to the aqueous ferrite nanoparticles dispersion where they adsorb onto particle surface. In order to prevent nanoparticle aggregation the polymers must contain two types of segments: an ionic one that grafts on the nanoparticle surface and a non-polar tail that extends into the solvent and ensures the colloidal stability by steric repulsion. Moreover, since the ionic segment is achieved through the deprotonation of carboxylic acids, the ionic strength and the $\mathrm{pH}$ of the medium should be controlled during the synthesis to adjust the nanoparticle charge, therefore allowing the surface complexation.

We have been working with materials based on magnetic ferrite nanoparticles dispersed in aqueous medium, known as electric double layered magnetic fluids (EDLMF) (Tourinho et al 1998). In a previous paper, we used electrochemical measurements to point out that the EDLMF system behaves as a mixture of acids: a strong one related to the bulk dispersion and a weak diprotic acid associated to the particle surface, which can be then functionalized with various chemical groups (Campos et al 2001). Furthermore, in the framework of the two-pK model, it has been possible to determine both the molar ratio of the nanoparticle surface sites as a function of $\mathrm{pH}$ and saturation value of the surface charge density. Actually, the intrinsic magnetic behaviour of the EDL-MF nanoparticles and their reactive surface make them particularly attractive as precursors for synthesis and design of magnetic nanosorbents for environmental applications. 
The focus of this work, in a first attempt, is to explore the particle surface-ligand complexation in order to propose an experimental procedure to develop a new type of magnetic nanosorbent for environmental applications composed by EDL-MF nanoparticles coated with PDMS. In this way, by using our experimental electrochemical approach, we first obtain the $\mathrm{pH}$ dependence of the surface charge density for a precursor EDL-MF based on manganese ferrite nanoparticles. Then, the speciation diagram for the surface sites is coupled with that of carboxylate-functional PDMS to analyse the mechanism of surface-PDMS complexation, allowing not only the theoretical prediction of the optimal $\mathrm{pH}$ for the synthesis but also the $\mathrm{pH}$ range for colloidal stability of the system.

\section{Experimental}

\subsection{Sample synthesis}

The EDL-MF samples under investigation are prepared as described before (Tourinho et al 1990). Manganese ferrite nanoparticles were synthesized by hydrothermal coprecipitation of aqueous solutions of manganese (II) and iron (III) chlorides in alkaline medium (methylamine). Next, the precipitate was washed and hydrothermally treated with a solution of iron (III) nitrate to avoid particle dissolution in acid medium. As a consequence, the chemical composition of the particles was no longer homogeneous and a core-shell model describing the particles as a core of stoichiometric spinel ferrite surrounded by a maghemite surface layer was recently proposed to account for the iron enrichment of the particle surface (Gomes et al 2008). Finally, the particles were peptized in acidic medium by adjustment of the ionic strength with nitric acid, leading to a stable sol of high quality. The chemical composition of the synthesized material was quantified by chemical analysis as dichromatometry titration and atomic absorption spectrometry.

\subsection{Structural and magnetic characterization}

The size determination and the structural characterization were investigated by room temperature X-ray powder diffraction (XRD) performed at the Brazilian Synchrotron source (Laboratório Nacional de Luz Síncrotron - LNLS) using the D12A-XRD1 beam line, monochromatized at $6.01 \mathrm{keV}(\lambda=2 \cdot 0633 \AA)$. Diffraction patterns were obtained typically within $20^{\circ} \leq 2 \theta \leq 130^{\circ}$ interval, with $0 \cdot 04^{\circ}$ step and $10 \mathrm{~s}$ counting time.

The magnetic measurements performed at $300 \mathrm{~K}$ and in fields up to $4 \times 10^{3} \mathrm{kA} / \mathrm{m}$ were achieved by using a superconducting quantum interference device (SQUID). The samples were studied in a concentration range and $\mathrm{pH}$ conditions where the interparticle interaction could be considered negligible.

\section{3 pH-dependent surface charge determination}

In order to determine the surface charge of our nanoparticles we have performed simultaneously potentiometricconductimetric titrations (Campos et al 2001) of $40 \mathrm{ml}$ of a magnetic colloid sample (volume fraction $\phi=1.5 \%$ ), under $\mathrm{CO}_{2}$-free atmosphere and using sodium hydroxide as titrant. The titrations were carried out with an electronic burette Metrohm 751 DOSIMAT. The potentiometric readings were performed with a pHmeter Metrohm 713 using a pH glass double-junction electrode while the conductivity was measured with a conductometer Metrohm 712 using a conductivity cell specially designed for colloidal dispersions.

\section{Results and discussion}

Figure 1 shows the X-rays pattern obtained for our EDLMF nanoparticle sample and exhibits several lines corresponding to the characteristic interplanar spacings (111), (220), (311), (400), (422), (511), (440), (620), (533) and (622) of the spinel structure.

The size of the cubic cell is found equal to $0.840 \mathrm{~nm}$ to be compared with the ASTM value equal to $0.849 \mathrm{~nm}$ for $\mathrm{MnFe}_{2} \mathrm{O}_{4}$ bulk material. The mean crystal size $d_{\mathrm{XR}}$ was deduced by means of the Scherrer formula. Using the width at half-maximum of the most intense diffraction line (311), we get $d_{\mathrm{XR}}=7 \cdot 3 \mathrm{~nm}$.

Figure 2 shows a magnetization curve of dilute samples, obtained at $300 \mathrm{~K}$, which exhibits a giant paramagnetic behaviour since at zero field, the magnetization is zero; as the applied field $H$ increases, the magnetization increases too, and no hysteresis is observed in the investigated range of field. The inset of figure 2 shows an initial susceptibility measurement showing that, at low field, the magnetization $M$ is proportional to the magnetic field.

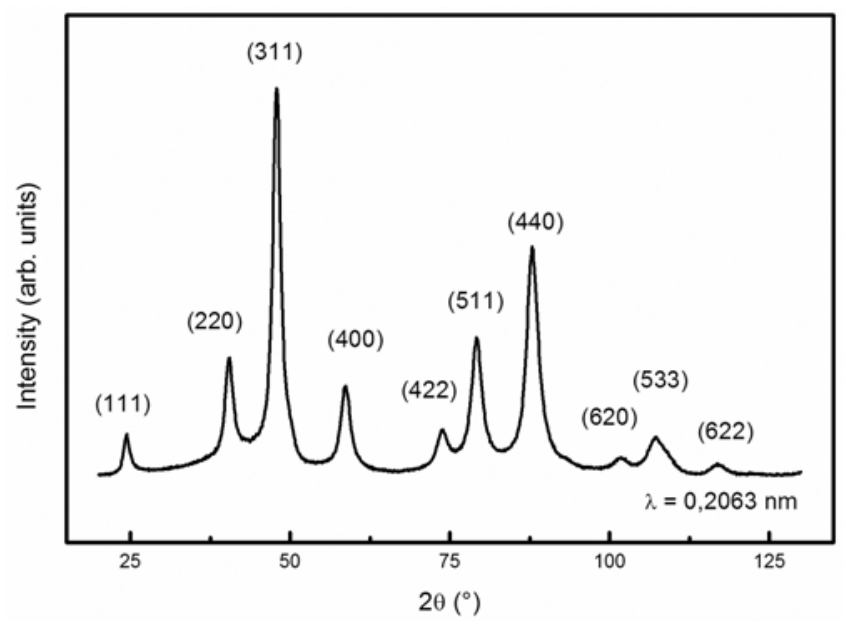

Figure 1. XRD pattern: the characteristic $(h k l)$ interplanar planes of the spinel structure are labeled. 
From the slope of the initial magnetic susceptibility curve, it is possible to calculate the dipolar interaction parameter $\gamma=0 \cdot 36$, a result that indicates (Gazeu et al 2003) that the magnetic particle-particle interactions are negligible. Then the magnetic behaviour of our EDL-MF can be compared to a Langevin model where the size distribution is taken into account by considering a log-normal volume-weighted superposition of the contributions of all different particles volume. The saturation magnetization of the magnetic particle and the size-distribution parameters as determined from the best fit of the magnetization curve are $m_{\mathrm{S}}=285 \mathrm{k} / \mathrm{Am}, d_{0}=6.4 \mathrm{~nm}$ and $s_{\mathrm{d}}=0.27$.

The results of the electrochemical measurements are depicted in figure 3 , which shows three distinct regions related first to the strong acid titration of the bulk

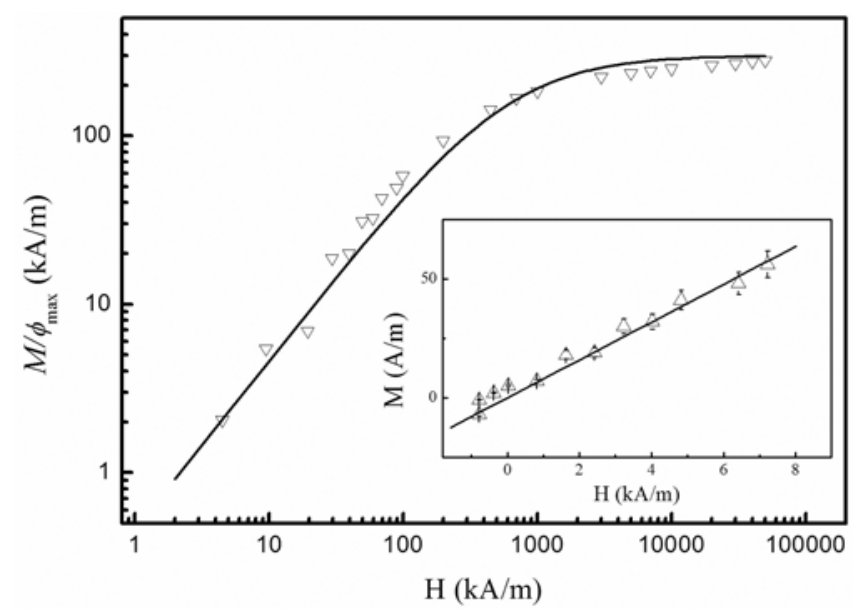

Figure 2. Room temperature magnetization curve for our sample; the full line is the best Langevin adjust. The inset displays the initial magnetic susceptibility and the full line is a linear fit.

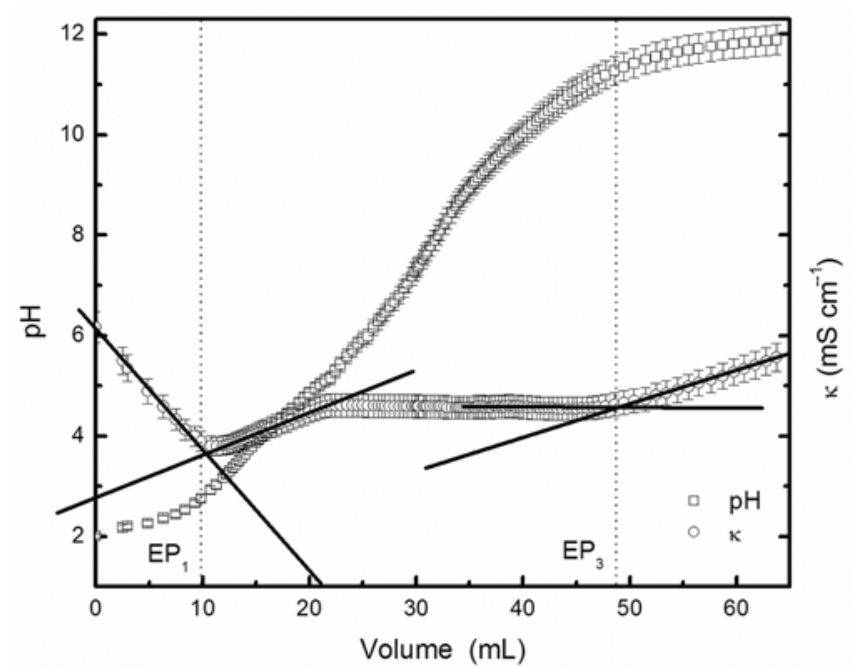

Figure 3. Simultaneously potentiometric and conductimetric titrations obtained for the magnetic fluid sample. dispersion, then to the nanoparticle surface neutralization and finally to the excess of the added base. The Brönsted acid-base behaviour of particle surface results from hydrolysis reactions, which lead to a $\mathrm{pH}$-dependent protonation/deprotonation process that has been described by using a two-pK model according to the following equilibria (Campos et al 2001):

$$
\begin{aligned}
& \mathrm{MOH}_{2}^{+}+\mathrm{H}_{2} \mathrm{O} \rightleftharpoons \stackrel{\mathrm{pK}_{1}}{\rightleftharpoons} \mathrm{MOH}+\mathrm{H}_{3} \mathrm{O}^{+} \text {, } \\
& \mathrm{MOH}+\mathrm{H}_{2} \mathrm{O} \stackrel{\mathrm{pK}_{2}}{\rightleftharpoons} \mathrm{MO}^{-}+\mathrm{H}_{3} \mathrm{O}^{+},
\end{aligned}
$$

where $M$ is the metal of the spinel type nanoparticles. From the determined equivalence points of the titration curves (Kildahl and Varco-Shea 1996), we calculated the concentration of surface sites and the $\mathrm{pK}$ values of the surface charging equilibrium process $\mathrm{pK}_{1}=4.8$ and $\mathrm{pK}_{2}=9 \cdot 8$. The modulus of the saturation value of the nanoparticle surface charge density was found equals to $0.23 \mathrm{Cm}^{-2}$, which corresponds to 240 surface sites

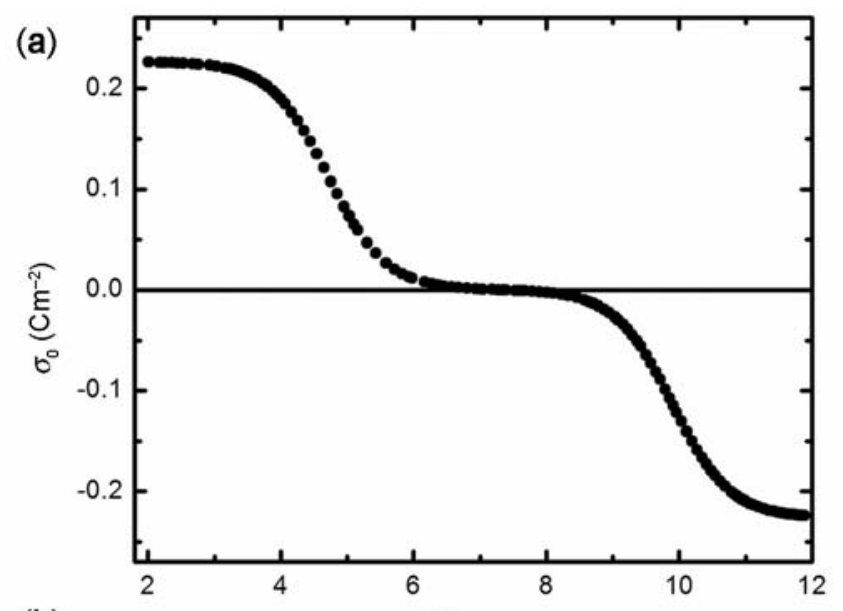

(b) $\mathrm{pH}$

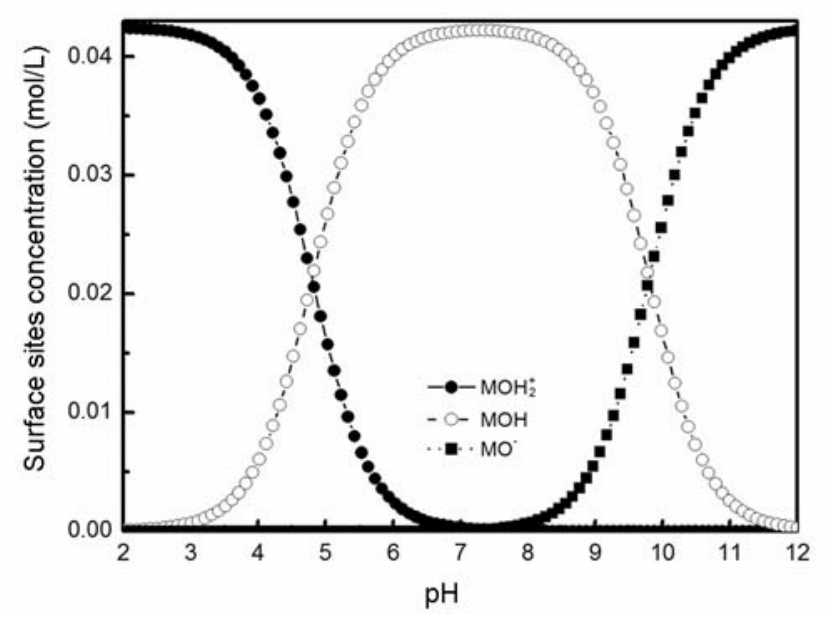

Figure 4. (a) $\mathrm{pH}$ dependence of the superficial density of charge for EDL-MF sample. (b) Speciation diagrams for nanoparticle surface sites. 
per particle or 1 charge per $0.7 \mathrm{~nm}^{2}$. The profile of the $\mathrm{pH}$ dependence of the surface charge density for the nanoparticles is shown in figure 4(a) and agrees with reported results (Campos et al 2001). For $\mathrm{pH}$ values lower than $4 \cdot 0$ and higher than 11.0 the particle surface tends to be charge-saturated, which ensure the colloidal stability of the magnetic sol for convenient ionic strength conditions. Around the neutral region the particle charge tends to zero and rapid coagulation can be evidenced (Campos et al 2009). The concentration of particle surface sites was plotted in figure 4(b) as a function of $\mathrm{pH}$. This speciation diagram gives a quantitative description of the particle surface, illustrating the domains of protonation of surface sites. For small $\mathrm{pH}$ ranges around $\mathrm{pK}$ values, figure 4(b) evidences the coexistence of charged and neutral sites.

The PDMS oligomers used in the magnetic nanosorbent preparation do not present chemical affinity for the nanoparticle surface. In this way, it has been used as a procedure that incorporates carboxyl groups on the PDMS through a polycondensation reaction of hydroxylterminated PDMS precursors with a dichlorosilane compound containing a tert-butyl-protected carboxylic acid group (Pribil 1982). The carboxyl-functional PDMS obtained, PDMS-COOH, $\mathrm{pK} \approx 4 \cdot 3$ (Mengistu et al 2006), can now complex the metal oxide nanoparticles according to the schematic equation of the complexation reaction:

$$
\equiv \mathrm{MOH}_{2}^{+}+\mathrm{PDMS}_{-\mathrm{COO}^{-}} \rightleftharpoons \equiv \mathrm{M}-\mathrm{OOC}-\mathrm{PDMS}+\mathrm{H}_{2} \mathrm{O},(3)
$$

where PDMS- $\mathrm{COO}^{-}$is the dissociated form of the carboxyl-functional PDMS. In fact, it is well known that carboxylate acid groups form remarkably stable chelates with many metal ions in solution (Batra et al 2006). Thus, the process of nanoparticle coating with carboxylfunctional PDMS oligomers should be controlled by the $\mathrm{pH}$, which also monitors the surface charge density.

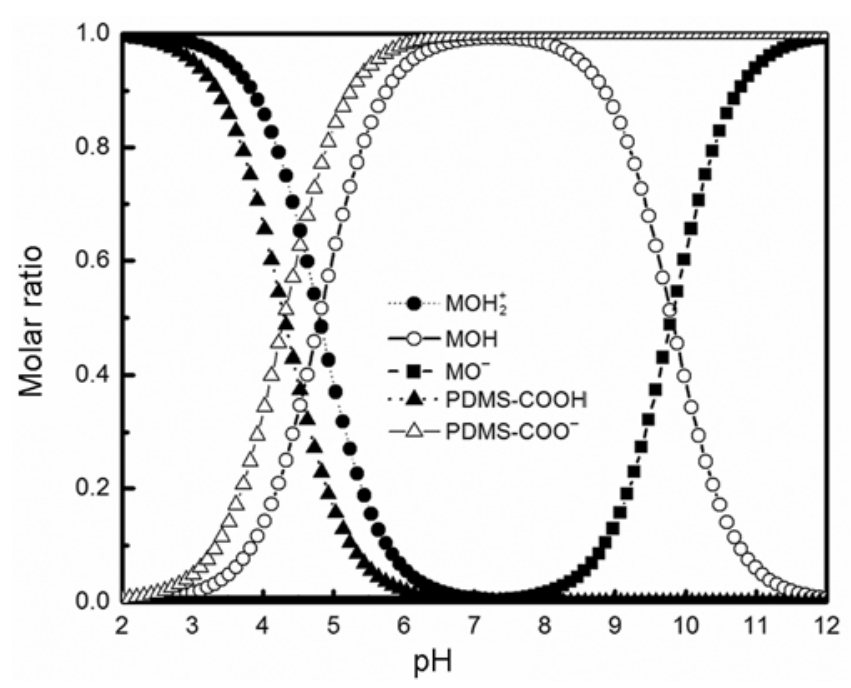

Figure 5. Coupled speciation diagrams of surface sites and carboxylate-functional PDMS ligands.
In this context, we plotted in figure 5 the coupled speciation diagrams of particle surface sites and carboxylfunctional PDMS. As can be seen, in alkaline medium, both nanoparticles and PDMS ligands are negatively charged and no complexation would occur. On the other hand, in acidic medium the particle surface is positively charged, allowing its functionalization. From these considerations, one can readily conclude that the optimal $\mathrm{pH}$ for nanoparticle-ligand complexation is around 4.5, where the carboxylic groups of PDMS ligands are mostly dissociated. Indeed, both the stoichiometry of the schematic reaction (3) combined with the species diagram of figure 5 allow us to deduce that the best chemical yield is obtained for this $\mathrm{pH}$ value. These theoretical predictions are in good agreement with experimental results involving the synthesis of polydimethylsiloxane-magnetite nanoparticle complexes for biomedical applications (Wilson et al 2005). The authors evidenced that the magnetite complexation was highly effective in acidic medium $(3 \leq \mathrm{pH} \leq 6)$, while for $\mathrm{pH}>8$ the magnetic complexes were not stable.

\section{Conclusions}

In this work, we focused on the environmental applications of magnetic ferrite nanoparticles. From a theoretical point of view, we explored the oxide particle-ligand complexation to improve the synthesis of PDMS complexes based on ferrite nanoparticles, which can be used as promising nanosorbents to remove organic pollutants from water by magnetic separation. From potentiometric and conductimetric titrations, we determined the surface charge density for EDL-MF precursor sample based on manganese ferrite nanoparticles according to the two-pK model. Then, the speciation diagram of the surface charged sites was coupled with that of the carboxylic-functional PDMS oligomers to analyse the mechanism of particle surface-ligand complexation. The obtained results showed that the carboxylate groups can bind the nanoparticle surface only in acid $\mathrm{pH}$ medium where the surface sites are positively charged, which confirms experimental recent reports. Our approach allowed a theoretical prediction of the optimal $\mathrm{pH}$ for ligandsurface complexation, therefore proving to be a very interesting tool in the magnetic nanosorbents developing. Finally, the next step of our investigation will concern the preparation of PDMS-COOH-coated magnetic nanosorbent, according to the parameters established in this work, and its characterization in terms of surface coverage, surface hydrophobicity and wettability, and pollutant adsorption capacity.

\section{Acknowledgements}

The authors thank the Brazilian Agencies (CAPES, CNPq, FINATEC and FAP-DF) for their financial 
support. We also acknowledge Legrand, from the Groupe de Physique de Solide of Universite Paris 6, for the SQUID measurements.

\section{References}

Apblett A W, Al-Fadul S M, Chehbouni M and Trad T 2001 Proc. 8th Int. Environ. Petroleum Consortium

Batra A, Cohen C and Duncan T M 2006 Macromolecules 392398

Campos A F C, Tourinho F A, Silva G J, Lara M C F L and Depeyrot J 2001 Eur. Phys. J. E6 29

Campos A F C, Marinho E P, Ferreira M A, Tourinho F A, Paula F L O and Depeyrot J 2009 Braz. J. Phys. 39230

Gazeau F, Boué F, Dubois E and Perzynski R 2003 J. Phys. Condens. Matter 15 S1305

Gomes J A, Sousa M H, Tourinho F A, Aquino R, Silva G J, Depeyrot J, Dubois E and Perzynsky R 2008 J. Phys. Chem. C112 6220
Kildahl N and Varco-Shea T 1996 Explorations in chemistry (New York : John Wiley \& Sons)

Konieczny K, Bodzek M and Panek D 2007 Desalination 223 344

Mayoa J T, Yavuza C, Yeanb S, Congb L, Shipleyb H, Yua W, Falknera J, Kanb A, Tomsonb M and Colvina V L 2007 Sci. Technol. Adv. Mater. 871

Mengistu T Z, Goel V, Horton J H and Morin S 2006 Langmuir 225301

Pribil R 1982 Applied complexometry (Oxford: Pergamon Press)

Savage N and Diallo M S 2005 J. Nanopart. Res. 7331

Tourinho F A, Depeyrot J, Silva G J and Lara M C F L 1998 Braz. J. Phys. 28413

Tourinho F A, Franck R and Massart R 1990 J. Mater. Sci. 25 3249

Tratnyek P G and Johnson R L 2006 Nanotoday 144

Wilson K S, Goff J D, Riffle J S, Harris L A and St Pierre T G 2005 Polymer Adv. Technol. 16200 